\title{
Improvement of Gradient Projection Algorithm for Nonlinear Programming
}

\author{
Dan Wang \\ Mathematics teaching and research section \\ Beijing Institute of Graphic Communication \\ BIGC \\ Beijing,China \\ wangdan@bigc.edu.cn
}

\begin{abstract}
The search direction by making use of the matrix LU decomposition gradient projection algorithm for nonlinear programming is given, the stability of sparse and thealgorithm of this method can maintain effective constraintmatrix, the algorithm can be applied to large sparsenonlinear optimization problem with linear constraints.
\end{abstract}

Keywords- lu decomposition, large scale sparse, nonlinear optimization.

\section{INTRODUCTION}

Consider $\min \{f(x) \mid x \in S\}$,

$S$ is a set defined by $S=\{x \mid A x=b, x \geq 0\}$,

$S_{+}=\{x \mid x \in S, x \succ 0\}$.

Structuring gradient direction $P_{A^{\perp}}$ in projection algorithm is the same as finding the answer of least-squares of overdetermined equations $A^{T} x=c$. Assume $A$ is Large matrix, since the $\mathrm{QR}$ decomposition may not keep the sparsity of large-scale, we cannot use QR decomposition to solve it. In order to solve the above problems, we consider using the matrix LU decomposition to construct descent direction.

\section{BASED ON THE STRUCTURE OF VERTICAL SPACE}

For a matrix $A_{m \times n}(m \succ n)$, assume the rank of $A$ is $n$,according to the theory of Gauss elimination[1],exist the matrix $M_{1}, M_{2}, \cdots, M_{n}$, subject to

note

$$
M_{n} \cdots M_{2} M_{1} A=\bar{U}=\left(\begin{array}{cccc}
* & * & \cdots & * \\
0 & * & \cdots & * \\
0 & 0 & \ddots & * \\
0 & 0 & \cdots & * \\
0 & 0 & \cdots & 0 \\
0 & 0 & \cdots & 0 \\
0 & 0 & \cdots & 0
\end{array}\right) \text {, }
$$

$M=M_{n} \cdots M_{2} M_{1}$,

so (1) induce
Yong-ming Zhang

Mathematics teaching and research section

Beijing Institute of Graphic Communication BIGC

Beijing,China

bjzym@bigc.edu.cn

$$
M A=\left(\begin{array}{cccc}
* & * & \cdots & * \\
0 & * & \cdots & * \\
0 & 0 & \ddots & * \\
0 & 0 & \cdots & * \\
0 & 0 & \cdots & 0 \\
0 & 0 & \cdots & 0 \\
0 & 0 & \cdots & 0
\end{array}\right)
$$

The (2) both sides of transposition, obtain $A^{T} M^{T}$

$$
=\left(\begin{array}{ccccccc}
* & 0 & 0 & 0 & 0 & \cdots & 0 \\
* & * & 0 & 0 & 0 & \cdots & 0 \\
\vdots & \vdots & \ddots & \vdots & 0 & \cdots & 0 \\
* & * & * & * & 0 & \cdots & 0
\end{array}\right)
$$

Inside $M_{1}, \mathrm{M}_{2}, \cdots, M_{n}$ are lower triangular matrix, so

$$
M^{T}=\left(M_{n} \cdots M_{2} M_{1}\right)^{T}=M_{1}{ }^{T} M_{2}{ }^{T} \cdots M_{n}{ }^{T}
$$

are upper triangular matrix.

Theorem 1.

Assume $\bar{U}=\left(u_{n+1}, u_{n+2}, \cdots, u_{m}\right)$, then $\bar{U}$ is a group of base on vertical subspace of $A$.

Proof.

Assume

$$
\begin{gathered}
M^{T}=\left(u_{1}, u_{2}, \cdots, u_{n}, \cdots, u_{m}\right), \\
\text { so }(3) \text { can be changed to } \\
A^{T}\left(u_{1}, u_{2}, \cdots, u_{n}, \cdots, u_{m}\right) \\
=\left(\begin{array}{ccccccc}
* & 0 & 0 & 0 & 0 & \cdots & 0 \\
* & * & 0 & 0 & 0 & \cdots & 0 \\
\vdots & \vdots & \ddots & \vdots & 0 & \cdots & 0 \\
* & * & * & * & 0 & \cdots & 0
\end{array}\right)
\end{gathered}
$$

Because the rank of $A$ is $n$,so the matrix 


$$
\left(\begin{array}{ccccccc}
* & 0 & 0 & 0 & 0 & \cdots & 0 \\
* & * & 0 & 0 & 0 & \cdots & 0 \\
\vdots & \vdots & \ddots & \vdots & 0 & \cdots & 0 \\
* & * & * & * & 0 & \cdots & 0
\end{array}\right)
$$

From the $(\mathrm{n}+1)$ th column is vector 0 .

so

$$
\begin{aligned}
& A^{T} u_{n+1}=0 \\
& A^{T} u_{m}=0
\end{aligned}
$$

$\bar{U}$ is a group of base on vertical subspace of A.

According to Theorem 1, we can obtain the corresponding algorithm to solve $\bar{U}$, denoted Algorithm 1 .

Because the effective constraint coefficient matrix of large-scale problems often have some sparse structures, we can make the constraint matrix become a banded structure by adjusting the operative constraint and the order of unknown sequence, so using algorithm 1 calculation $\bar{U}$, it can not only looser the sparsity of the constraint matrix requirements, but it can also decrease the storage capacity of new method than the usual LU decomposition.

\section{THE GRADIENT PROJECTION ALGORITHM FOR NONLINEAR PROGRAMMING}

Considering the nonlinear programming problem $\min \{f(x) \mid x \in S\}$,

$S$ is a set defined by

$$
\begin{aligned}
& S=\{x \mid A x=b, x \geq 0\}, \\
& S_{+}=\{x \mid x \in S, x \succ 0\}
\end{aligned}
$$

Algorithm 2

Step 1. Set the initial point $x^{0} \in S_{+}, k=0$.

Step 2. Using $x^{k}$ the definition of diagonal matrix $X=\operatorname{diag}\left(x_{1}^{k}, \cdots, x_{n}^{k}\right)$,

do transform

$$
\begin{gathered}
\bar{x}=X^{-1} x, \\
\bar{c}=X C, \\
\bar{A}=A X
\end{gathered}
$$

Step 3. Using algorithm 1 to solve u, u satisfy: $\bar{A}^{T} u=0$.

Step 4. Solve the gradient projection direction:

$$
\begin{gathered}
d f=d f G * \bar{x}+d f b \\
\bar{c}_{p}=-u u^{T} d f \\
\bar{d}=\frac{1}{\left\|\bar{c}_{p}\right\|} \bar{c}_{p}
\end{gathered}
$$

Step 5. Iterative:

$$
\bar{x}^{-k+1}=e+\alpha \bar{d}, 0<\alpha<1
$$

$$
x^{k+1}=X^{-k+1} .
$$

Step 6. If the termination condition is satisfied, then the end; otherwise $k:=k+1$, return step 2 .

\section{EXAMPLE}

Example 1:

solve the nonlinear programming

$$
\begin{aligned}
& \min \left(x_{2}^{2}+x_{4}^{2}+\cdots+x_{98}^{2}+x_{100}^{2}\right) \\
& \quad \text { s.t. } x_{i}+x_{i+1}=0.1, i=1,2, \cdots, 100 \\
& x_{i} \geq 0, i=1,2, \cdots, 101
\end{aligned}
$$

Obviously, the optimal objective function value of the linear programming is 0 , one of it is:

From the initial point of

$$
\begin{aligned}
x_{2 i+1} & =0.1, i=0,1,2, \cdots, 50, \\
x_{2 i} & =0.0, i=1,2, \cdots, 50 .
\end{aligned}
$$

$x_{1}=x_{2}=\cdots=x_{100}=x_{101}=0.05$,

after 311 iterations, the optimalvalue of 4.8640e-013.

Example 2:

solve the nonlinear programming

$$
\begin{gathered}
\min \left(x_{3}^{2}+x_{6}^{2}+\cdots+x_{99}^{2}+x_{102}^{2}\right) \\
\text { s.t. } x_{i}+x_{i+1}+x_{i+2}=0.6, \\
i=1,2, \cdots, 100 \\
x_{i} \geq 0, i=1,2, \cdots, 102
\end{gathered}
$$

Obviously, the optimal objective function value of the linear programming is 0 , one of it is:

$$
\begin{aligned}
& \quad x_{3 i+1}=0.4 \quad, \quad x_{3 i+2}=0.2, \quad x_{3 i+3}=0.0, \\
& i=1,2, \cdots, 33 \text {. } \\
& \text { From the initial point of }
\end{aligned}
$$

$x_{1}=x_{2}=\cdots=x_{100}=x_{101}=0.2$,

after 306 iterations, the optimalvalue of 4.7609e-013.

\section{ACKNOWLEDGMENT}

Wang Dan and Zhang Yong-ming thank the Support of Beijing high school youth talent plan (YETP1471) and Beijing Institute of Graphic Communication key projects (22150114021).

\section{REFERENCES}

[1] Karmarkar N.K.,A new polynomial-time algorithm for linear programming.Combinatorica,4:373-395, 1984.

[2] D.Goldfarb \& Liu, An O( $\left.n^{3} \mathrm{~L}\right)$ Primal Interior Point Algorithm for Convex Quadric Programming. Mathematical Programming, Vol. 49, 325-340, 1991.

[3] CHEN Z \& DENG N Y. Some algorithms for the convex guadratic problem via the ABS approach[J].Optimization Methods and Software. 8(2): 157-170, 1997.

[4] Li Qing-yang, Wang Neng-chao \& Yi Da-yi, Numerical analysis. Huazhong University of science and Technology Press,1999.

[5] Chen Zhi \& Gao Lv-duan, An Algorithm for Solving Large-scale Sparse Group of Linear Equations. JOURNAL OF BEIJING POLYTECHMC UMVERSITY, Vo1.27 Sep.N0.3,2001. 